\title{
ESCENARIOS DE SALINIDAD DEL SUELO POR CAMBIO DEL SISTEMA DE RIEGO EN LA ZONA DE REGADÍO TRADICIONAL DE LA VEGA BAJA DEL SEGURA (ALICANTE)
}

\author{
Visconti Reluy, F. (1), de Paz Bécares, J.M. (2) (P)
}

\begin{abstract}
1 Titulado Superior, Instituto Valenciano de Investigaciones Agrarias, Centro para el Desarrollo de la Agricultura Sostenible, visconti fer@gva.es

${ }^{2}$ Colaborador Científico Adjunto, Instituto Valenciano de Investigaciones Agrarias, Centro para el Desarrollo de la Agricultura Sostenible, depaz jos@gva.es
\end{abstract}

\section{Resumen}

La modernización proyectada para el sistema de riego en la zona de regadío tradicional de la Vega Baja del Segura genera cierta oposición en los agricultores. Esto es debido al reconocido efecto sobre el lavado de las sales de los suelos que tiene el regar a manta con un cierto exceso de agua. En el presente trabajo se presenta la simulación de varios escenarios de cambio del sistema de riego y sus efectos sobre la salinidad de los retornos de riego y los suelos en la parte final de la zona. Esta parte final está constituida por tres jurisdicciones de riego: Juzgado Privativo de Aguas del Azud de Alfeitamí, Sindicato Central de Aguas de Dolores y Sindicato General de los Carrizales. El agua del río Segura se deriva en el azud de Alfeitamí y se riega la primera jurisdicción y luego secuencialmente las otras dos aprovechando cada una de estas los retornos de riego de la anterior. Para realizar las simulaciones se ha desarrollado un modelo hidrológico sencillo del funcionamiento de este sistema, y se ha integrado el modelo de salinidad de suelos SALTIRSOIL dentro de este. De acuerdo con los resultados de las simulaciones el cambio del sistema de riego en si ocasionaría pocos cambios: salinidad más baja en JPA Azud de Alfeitamí, igual en SCA Dolores, y más alta en SG Carrizales. No obstante, es esperable que el cambio del actual sistema de regadío a uno de mayor eficiencia dé lugar a una disminución proporcional de la dotación de riego para toda zona, y en este supuesto, sí que ocurrirían cambios importantes en la salinidad de los suelos. En concreto, ocurriría un aumento cada vez mayor de la salinidad en la secuencia de reutilización del agua. Por esto se recomienda elaborar un modelo completo de la zona de regadío tradicional de la Vega Baja del Segura como banco de pruebas para anticipar los efectos de varias alternativas de modernización del regadío en el área.

\section{Abstract}

The modernization that is planned for the irrigation system in the traditional irrigation area of the Vega Baja del Segura faces some opposition in the farmers. This is due to the recognized effect that surface irrigation with a certain water excess has for flushing out the soil salts. The present work presents the simulation of several scenarios of change of the irrigation system and their effects on the salinity of the irrigation return flows and soils in the final part of the zone. This final part is constituted by three irrigation jurisdictions: Juzgado Privativo de Aguas del Azud de Alfeitamí, Sindicato Central de Aguas de Dolores and Sindicato General de los Carrizales. The water of the Segura river is diverted in the Alfeitamí dam and it irrigates the first jurisdiction and then, sequentially, the other two in which each one of them makes the most of the irrigation return flows from the previous one. In order to carry out the simulations, a simple hydrological model of this system has been developed, and the SALTIRSOIL soil salinity model has been integrated into it. According to the results of the simulations, the change of te irrigation system itself would result in few changes: lower 
salinity in JPA Azud de Alfeitamí, equal in SCA Dolores, and higher in SG Carrizales. However, it is expected that the change from the present irrigation system to one of higher efficiency will result in a proportional reduction of the irrigation supply for the entire zone, and in this case, it would certainly lead to important changes in the salinity of the soils. Specifically, soil salinity would increase in the water reuse sequence. For this reason, the development of a complete model of the traditional irrigation area of the Vega Baja del Segura is recommended as a test bed to foresee the effects of several modernization alternatives for the irrigation system in the zone.

\section{Introducción}

La zona de regadío tradicional de la Vega Baja del Segura se localiza principalmente en la comarca homónima, y en menor medida, en la del Bajo Vinalopó, ambas en el extremo sur de la provincia de Alicante. El origen de este regadío se remonta al menos al siglo VIII (Gea, 1997), y actualmente comprende una superficie total de 23.391 ha de las cuales tan solo el $66 \%$ se riegan efectivamente cada año (CHS, 2015).

Este regadío tradicional está organizado en 18 jurisdicciones de riego que derivan el agua del río Segura mediante 8 azudes distribuidos a lo largo de su cauce en la Vega Baja. De estos azudes parten las acequias mayores, las cuales se ramifican en otras menores, y estas a su vez en otras aún menores, de tal modo que el agua llega hasta cada una de las parcelas de la zona. Los retornos de riego de cada parcela, es decir sobrantes de riego más aguas de drenaje, son evacuados mediante unos canales llamados escorredores situados al final de cada parcela. Estos canales se unen para formar otros mayores, y estos a su vez otros aún mayores llamados azarbes que conducen los retornos de riego de cada jurisdicción o bien hacia el río para su vertido, o bien hacia otra jurisdicción para su aprovechamiento como agua de riego (Melgarejo et al., 2013). Este sistema de riego y avenamiento donde los retornos son reutilizados, ya sea mezclados de nuevo con las aguas del río, ya sea directamente, se caracteriza por i) un elevado grado de eficiencia de uso del agua disponible en la zona, ii) el mantenimiento del nivel freático suficientemente alejado de la superficie del suelo, y iii) el mantenimiento de la salinidad de los suelos dentro de un margen adecuado para el cultivo.

A pesar de estas características favorables, el regadío tradicional de la Vega Baja del Segura también presenta deficiencias (Rocamora et al., 2016). Así que desde finales del siglo XX se viene proyectando su modernización, concretada en la actualidad en el "Plan de Obras de Modernización de Regadíos en la comarca de la Vega Baja" de la Generalitat Valenciana, cuya actuación principal gira en torno al cambio del riego de superficie a localizado (GVA, 2016). La mayor eficiencia de uso del agua que este sistema presenta frente al tradicional es el principal argumento y motivación para cambiarlo (Abadía et al., 1999). No obstante, en la cultura huertana de la Vega Baja el riego en exceso tal y como se hace en el regadío tradicional no es visto como un completo desperdicio de agua, sino como necesario para lavar las sales de los suelos. Esta idea de antiguo arraigo, pues ya fue documentada por Cavanilles (1797), ha sido refrendada por la ciencia moderna de tal modo que la práctica de regar con un cierto exceso de agua junto con un drenaje adecuado de la misma es universalmente reconocida como necesaria para mantener controlada la salinidad de los suelos de regadío (USSL Staff, 1954; Ayers y Westcot, 1985; Corwin et al., 2007).

El cambio del sistema de riego tendría consecuencias sobre la dotación de agua que la Confederación Hidrográfica del Segura (CHS) planifica para la zona de regadío tradicional de la Vega Baja, y en consecuencia, sobre el control de la salinidad de los suelos. Actualmente la demanda neta de agua de riego de la zona se calcula multiplicando la superficie media cultivada anualmente con cada tipo de cultivo por su evapotranspiración estimada (CHS, 2015), y se resta la precipitación. Para calcular la demanda bruta, la demanda neta se divide entre el producto de los tres coeficientes de eficiencia de riego 
formulados por el ICID/ILRI: conducción, distribución y aplicación (Bos y Nugteren, 1990). Al riego por superficie la CHS le asigna un coeficiente de eficiencia de aplicación de 0.70 , mientras que al riego localizado le asigna uno de 0.95. En consecuencia, debido al predominio del riego por superficie en un $90 \%$ de la zona, la eficiencia media de aplicación actual es 0.725 . Según este modelo la transformación completa del regadío de superficie a localizado en la zona se traduciría en una eficiencia media de aplicación de 0.939, la cual podría ocasionar una notable disminución de su dotación de riego en futuros planes hidrológicos de la demarcación del Segura. Como se ve el modelo utilizado por la CHS no tiene en cuenta la necesidad de lavar sales y, en consecuencia, no considera qué consecuencias tendría el cambio del sistema de riego sobre el control de la salinidad en suelos donde el agua de riego, además, nunca baja de $2 \mathrm{dS} / \mathrm{m}$. Esto genera resistencia en los agricultores a una modernización centrada en el cambio del riego de inundación a localizado (Rocamora et al., 2016). En lugar de esta solución, se aboga por una modernización basada en el mantenimiento y mejora del secular sistema existente.

La utilización de modelos de simulación que tengan en cuenta la salinidad para planificar el riego en zonas con clima de árido a semiárido y donde además, se utilizan aguas salinas como es el caso de la Vega Baja del Segura, es una práctica repetidamente recomendada (Tanji y Kielen, 2002, Corwin et al., 2007). Los usuarios pueden elegir entre diferentes modelos que exhiben diferentes grados de complejidad y flexibilidad. Así existen modelos extremadamente sencillos, y no obstante útiles, como los tradicionales LR (Rhoades, 1974) y FAO (Ayers y Westcot, 1985), concretados, respectivamente, en una sola o varias ecuaciones. A continuación está WATSUIT, el cual es una evolución de los anteriores incorporando, además, un modelo químico sencillo (Rhoades et al., 1992). Finalmente, en esta línea de desarrollo de modelos de estado estacionario, está SALTIRSOIL (Visconti y de Paz, 2016), que ha sido validado en varios entornos de regadío en la Comunidad Valenciana y con el cual se puede realizar una planificación mensual del riego. El modelo SALTIRSOIL puede ser, o bien usado directamente on-line en Internet (http://agrosal.ivia.es/), o bien se puede descargar (http://www.uv.es/ fervisre/) y utilizarlo uno mismo en su ordenador. El uso del modelo SALTIRSOIL acoplado con modelos hidrológicos sencillos que describan lo esencial del funcionamiento del regadío tradicional de la Vega Baja del Segura no se ha hecho, y no obstante, tiene notable interés para simular qué ocurriría como consecuencia de un hipotético cambio del sistema de riego de superficie a localizado en la zona.

\section{Objetivos}

En el presente trabajo se ha elaborado un modelo que describe el funcionamiento hidrológico básico de las tres últimas jurisdicciones de riego dentro de la zona de regadío tradicional de la Vega Baja del Segura. Estas jurisdicciones son el Juzgado Privativo de Aguas del Azud de Alfeitamí (JPA Azud de Alfeitamí), el Sindicato Central de Aguas de Dolores (SCA Dolores) y el Sindicato General de los Carrizales (SG Carrizales). Este modelo hidrológico se ha acoplado con el modelo SALTIRSOIL para estimar qué consecuencias tendría el cambio del sistema de riego de superficie a localizado en la zona formada por estas tres jurisdicciones. Fundamentalmente se han estudiado los cambios que ocurrirían en el caudal de los retornos de riego y su salinidad, y en la salinidad de los suelos.

\section{Materiales y Métodos}

Se elaboró un modelo hidrológico sencillo que describe el funcionamiento de la parte final del sistema de riego y drenaje de la zona de regadío tradicional de la Vega Baja del Segura, la cual está conformada por el JPA Azud de Alfeitamí, el SCA Dolores y el SG Carrizales. Esta última jurisdicción engloba parte del Parque Natural de las Salinas de Santa Pola (Fig. 1). 


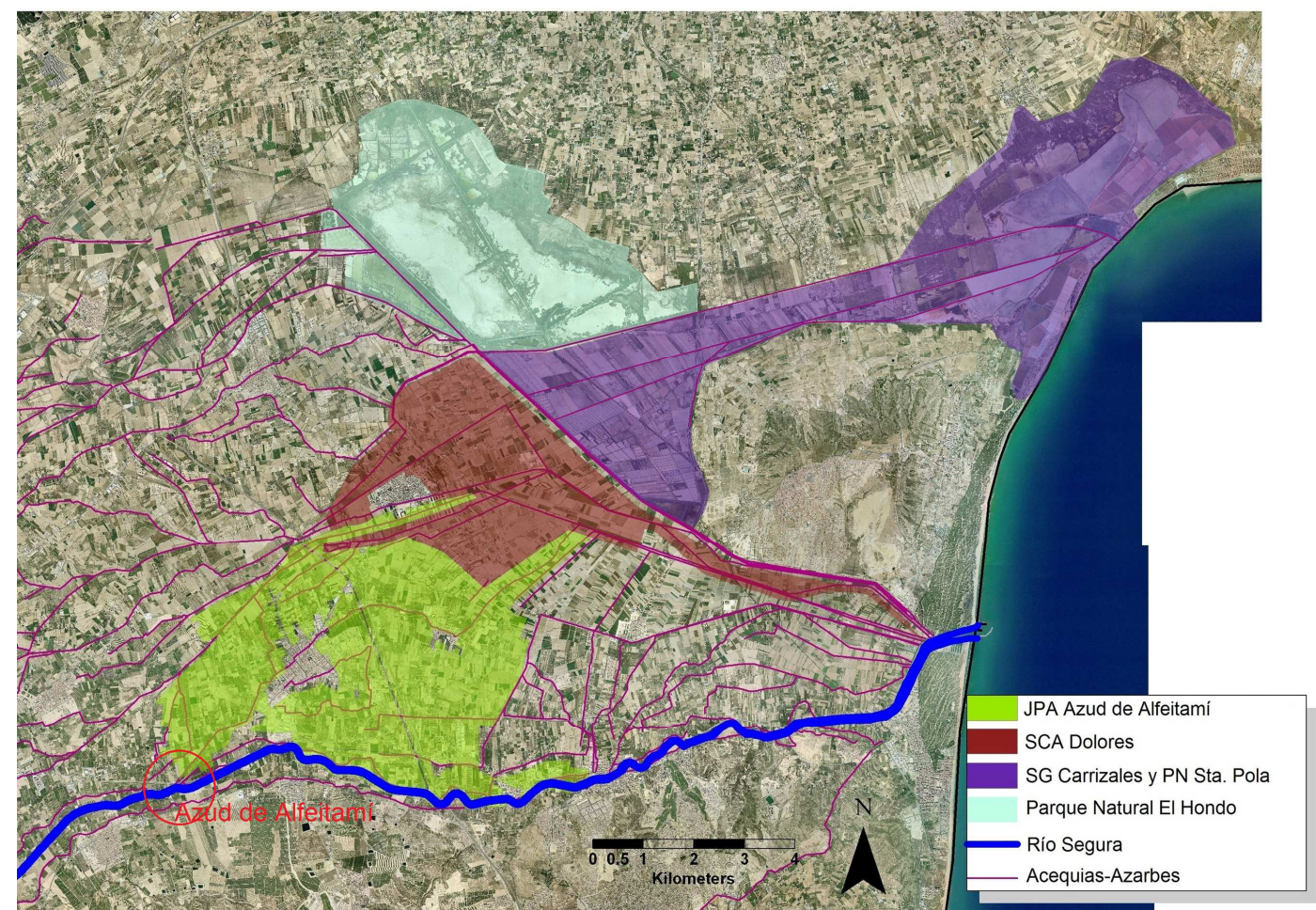

Figura 1. Sistema de riego y drenaje conformado por las tres jurisdicciones de riego en la parte final de la zona de regadío tradicional de la Vega Baja del Segura

La forma de riego y aprovechamiento del agua en esta zona consiste en i) la derivación del agua del río Segura en el azud de Alfeitamí, ii) el riego de esta primera jurisdicción (JPA Azud de Alfeitamí), iii) la reunión de sobrantes de riego y drenajes para conformar los retornos de riego de esta primera jurisdicción y cesión a la segunda jurisdicción, iv) el riego de la segunda jurisdicción (SCA Dolores) con dichos retornos de riego, v) la reunión de sobrantes de riego y drenajes de esta segunda jurisdicción para conformar sus retornos de riego y cesión a la tercera jurisdicción, vi) el riego de la tercera jurisdicción (SG Carrizales) con dichos retornos de riego y vii) la reunión de sobrantes de riego y drenajes para conformar los retornos de riego de esta tercera jurisdicción y cesión para otros usos entre los que se cuentan fundamentalmente los ecológicos en el Parque Natural de las Salinas de Santa Pola (Fig. 2 y Fig. 3).

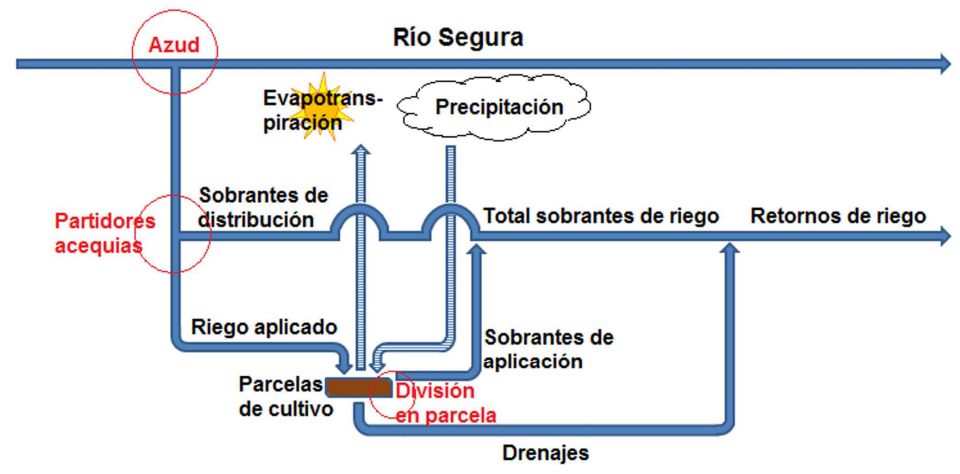

Figura 2. Modelo hidrológico de la jurisdicción de riego del JPA Azud de Alfeitamí, la cual deriva el agua del río Segura en el azud del mismo nombre, riega con dicha agua, reúne los sobrantes de riego y drenajes, y los cede a la jurisdicción siguiente para su aprovechamiento como agua de riego. 


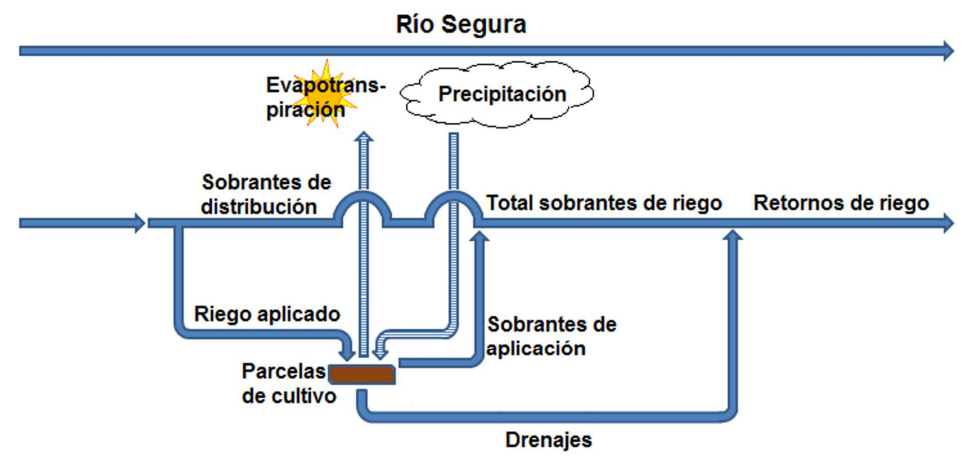

Figura 3. Modelo hidrológico de las jurisdicciones de riego del SCA Dolores y del SC Carrizales, las cuales riegan de los retornos de riego de la anterior jurisdicción, reúnen los sobrantes de riego y drenajes y los ceden a la siguiente jurisdicción para su aprovechamiento como agua de riego en el caso de SCA Dolores y para, fundamentalmente, un último aprovechamiento ecológico en el Parque Natural de las Salinas de Santa Pola en el caso de SG Carrizales.

Se integró el modelo SALTIRSOIL con este modelo hidrológico sencillo para simular la salinidad de los suelos de regadío en cada una de las jurisdicciones de riego, así como para simular la salinidad de sus aguas de drenaje. Asimismo, se utilizó el modelo SALSOLCHEM para simular la salinidad que tendrían las aguas de mezcla de drenaje y sobrantes de riego, las cuales constituyen los retornos de riego.

En la Fig. 2 en rojo se han destacado los tres puntos de partición de aguas dentro de la jurisdicción de riego del JPA Azud de Alfeitamí: i) el azud que separa el agua que sigue circulando por el río Segura del agua que se distribuye al sistema de riego conformado por las tres jurisdicciones, ii) los partidores de distribución en las acequias de riego que separan el agua aplicada en el riego en las parcelas de los sobrantes de distribución, y iii) la división de aguas que ocurre dentro de cada parcela y que separa, por un lado, la que infiltra en el suelo, y por otro lado, la que escurre por la superficie (escorrentía) y a través de los macroporos del suelo (flujos preferenciales), y que constituyen los sobrantes de aplicación.

Tabla 1. Características para la planificación hidrológica actual de las tres jurisdicciones de riego en la parte final de la zona de regadío tradicional de la Vega Baja del Segura (CHS, 2015)

\begin{tabular}{|c|c|c|c|c|c|c|c|}
\hline \multirow[b]{2}{*}{ Jurisdicción } & \multirow{2}{*}{$\begin{array}{l}\text { Superficie } \\
\text { bruta / ha }\end{array}$} & \multirow{2}{*}{$\begin{array}{l}\text { Superficie } \\
\text { neta / ha }\end{array}$} & \multirow{2}{*}{$\begin{array}{l}\text { Demanda } \\
\text { neta / } \\
\mathrm{m}^{3} \mathrm{ha}^{-1} \text { año }^{-1}\end{array}$} & \multirow[b]{2}{*}{ CD } & \multirow[b]{2}{*}{ CA } & \multicolumn{2}{|c|}{ Demanda bruta } \\
\hline & & & & & & $\begin{array}{l}\mathbf{m}^{3} \mathbf{h a}^{-1} \\
\text { año }^{-1}\end{array}$ & $\begin{array}{l}\mathbf{h m}^{3} \\
a^{n} 0^{-1}\end{array}$ \\
\hline JPF & 2 & 18 & 3950 & 0.890 & 0.725 & 6123 & 11.1 \\
\hline SCA & 14 & $-u$ & 3950 & 0.8 & 0.7 & 6123 & 6.1 \\
\hline SG Carrizales & 1508 & 997 & 3950 & 0.890 & 0.725 & 6123 & 6.1 \\
\hline TOTAL & 5732 & 3791 & 3950 & 0.890 & 0.725 & 6123 & 23.2 \\
\hline
\end{tabular}

Para cada uno de los tres puntos de partición de aguas en el sistema se define un coeficiente de eficiencia o reparto. En el caso del azud este coeficiente define la dotación de agua para el sistema conformado por las tres jurisdicciones que riegan a partir de dicho punto. Su valor es calculado por la CHS en función de las necesidades de riego y la eficiencia tanto del sistema de distribución como del sistema de riego, y en el último Plan Hidrológico de la Demarcación del Segura presenta un valor de 0.486. Esto significa que un $48.6 \%$ del caudal anual del Segura justo antes del azud de Alfeitamí se deriva para el regadío de estas tres jurisdicciones de riego. Esto hace, teniendo en cuenta el caudal medio anual del río Segura antes del azud durante el trienio 2013-2016, el cual ascendió a 47.8 $\mathrm{hm}^{3} /$ año, una dotación de $23.2 \mathrm{hm}^{3} /$ año para regar una superficie neta de 3791 ha (Tabla 1). El coeficiente de distribución (CD) define las pérdidas de agua desde que entra en la jurisdicción hasta que alcanza las parcelas. Su valor depende del sistema de distribución, 
que en el caso de las acequias se estima en 0.890 , es decir, la CHS estima que un $11 \%$ en promedio del agua que entra en cada jurisdicción se pierde antes de alcanzar las parcelas. Finalmente el coeficiente de aplicación (CA) define el reparto de agua entre la que efectivamente infiltra en el suelo y la que contribuye a la escorrentía y flujos preferenciales. Su valor depende de la eficiencia del sistema de riego presentando valores de 0.7 para sistemas de riego por inundación y de 0.95 para sistemas de riego localizado.

La modernización del sistema de regadío conllevaría cambios tanto en el coeficiente de dotación de riego como en los coeficientes de distribución (CD) y aplicación (CA). En el presente trabajo se ha simulado qué efecto sobre la cantidad y calidad de las aguas que circulan por las tres jurisdicciones tendrían los cambios de estos coeficientes, en particular, los cambios en el CA y en el coeficiente de dotación de agua en el azud. En particular se estudió cómo cambiaría el CA desde el actual 0.725 hasta el previsible 0.939 , y el coeficiente de dotación desde el actual 0.486 hasta el previsible 0.375 . Los efectos que ocasionarían los cambios en el CD no se han estudiado para no complicar en exceso el análisis. Debe destacarse también que la jurisdicción de SG Carrizales recibe también los retornos de riego de la jurisdicción del Sindicato de Riegos de Catral (SRC), los cuales se utilizan también para riego. Este hecho no se ha considerado en el presente trabajo para no complicarlo en exceso. En concreto se simularon seis escenarios de modernización del regadío (Tabla 2 ).

Tabla 2. Escenarios de simulación del cambio del riego por inundación a localizado en las comunidades de regantes que usan el agua derivada del Segura en el azud de Alfeitamí

\begin{tabular}{|l|l|l|}
\hline Escenario & $\begin{array}{l}\text { Comunidades de regantes donde se implementa el } \\
\text { riego localizado* }\end{array}$ & $\begin{array}{l}\text { Dotación de riego en el } \\
\text { azud }\end{array}$ \\
\hline 1 & JPA Azud de Alfeitamí & Igual \\
\hline 2 & JPA Azud de Alfeitamí & Disminución proporcional \\
\hline 3 & JPA Azud de Alfeitamí, SCA Dolores & Igual \\
\hline 4 & JPA Azud de Alfeitamí, SCA Dolores & Disminución proporcional \\
\hline 5 & JPA Azud de Alfeitamí, SCA Dolores, SG Carrizales & Igual \\
\hline 6 & JPA Azud de Alfeitamí, SCA Dolores, SG Carrizales & Disminución proporcional \\
\hline
\end{tabular}

Tabla 3. Tipos de cultivos y superficies ocupadas en la zona de regadío tradicional de la Vega Baja del Segura (CHS, 2015)

\begin{tabular}{|l|r|}
\hline Cultivo & Porcentaje superficie \\
\hline Cereales de invierno & 7,0 \\
\hline Tubérculos (patata) & 2,0 \\
\hline Alfalfa & 0,2 \\
\hline Hortícolas protegido & 0,2 \\
\hline Hortícolas aire libre & 41,6 \\
\hline Cítricos & 45,1 \\
\hline Frutales no cítrico & 1,0 \\
\hline Olivar & 2,9 \\
\hline
\end{tabular}

Para realizar las simulaciones de la salinidad de los suelos y los drenajes con el modelo SALTIRSOIL se supuso un cultivo homogéneo en las tres jurisdicciones de riego. Este cultivo homogéneo surge como combinación de los cultivos que se desarrollan en la zona de regadío tradicional de la Vega Baja del Segura en proporción a la superficie que ocupan en la misma (Tabla 3). Así se calcularon los coeficientes basales de cultivo, las profundidades de enraizamiento y las áreas sombreadas para cada mes del año, datos necesarios para llevar a cabo las simulaciones con SALTIRSOIL (Tabla 4). La dotación de riego se distribuyó a la zona en proporción a la demanda de agua calculada como producto del coeficiente de cultivo por la evapotranspiración de referencia del año promedio 20002016 en la estación SIAR de Almoradí, la cual se puede considerar representativa de las tres jurisdicciones de riego (Tabla 5). Se usaron también datos de precipitación del año promedio 2000-2016 en la estación SIAR de Almoradí (Tabla 5). Para el suelo se tomó un 
perfil tipo de la zona (Tabla 6), y se calculó la salinidad y el balance de agua hasta $60 \mathrm{~cm}$. Para el agua se tomó la composición promedio del Segura en la zona del azud (Tabla 7).

Tabla 4. Coeficientes basales de cultivo $(\mathrm{Kcb})$, profundidad de enraizamiento (Prof. R.) y área sombreada (Área Som.) por el cultivo homogéneo equivalente de la zona de regadío tradicional de la Vega Baja del Segura

\begin{tabular}{|c|c|c|c|c|c|c|c|c|c|c|c|c|}
\hline \multirow{2}{*}{ Propiedad } & \multicolumn{12}{|c|}{ Mes } \\
\hline & Ene & Feb & Mar & Abr & May & Jun & Jul & Ago & Sep & Oct & Nov & Dic \\
\hline $\mathrm{K}$ & 0,52 & 0,52 & 0,53 & 0,65 & 0,64 & 0,56 & 0,51 & 0,43 & 0,48 & 0,51 & 0,55 & 0,67 \\
\hline Prof. R. & 53 & 53 & 56 & 60 & 55 & 55 & 53 & 54 & 51 & 56 & 52 & 54 \\
\hline Área Som.(\%) & 48 & 48 & 50 & 54 & 50 & 50 & 46 & 46 & 47 & 52 & 50 & 53 \\
\hline
\end{tabular}

Tabla 5. Evapotranspiración de referencia $\left(E T_{0}\right)$ para el periodo 2000-2016 y porcentaje de la dotación de riego anual usada cada mes $\left(R / R_{\text {anual }}\right)$ en la zona de regadío tradicional de la Vega Baja del Segura. Elaborado a partir de los datos de la estación SIAR de Almoradí para el periodo 20002016.

\begin{tabular}{|c|c|c|c|c|c|c|c|c|c|c|c|c|}
\hline \multirow{2}{*}{ Propiedad } & \multicolumn{12}{|c|}{ Mes } \\
\hline & Ene & Feb & Mar & Abr & May & Jun & Jul & Ago & Sep & Oct & Nov & Dic \\
\hline & 47 & 59 & 89 & 112 & 140 & 159 & 169 & 152 & 109 & 76 & 51 & 40 \\
\hline & 2 & 13 & 2 & 35 & 22 & 10 & 2 & $t$ & 32 & 35 & 43 & 40 \\
\hline $\mathrm{R} / \mathrm{R}_{\text {anual }}(\%)$ & 3,8 & 4,7 & 7,3 & 11,1 & 13,8 & 13,7 & 13,1 & 10,1 & 8,0 & 6,0 & 4,3 & 4,1 \\
\hline
\end{tabular}

Tabla 6. Propiedades del suelo tipo Vega Baja*

\begin{tabular}{|c|c|c|c|c|c|c|c|c|c|c|c|}
\hline № & $\begin{array}{l}\text { Lím. } \\
\text { Sup. } \\
\text { / cm }\end{array}$ & $\begin{array}{l}\text { Lím. } \\
\text { Inf. I } \\
\text { cm }\end{array}$ & $\begin{array}{l}\mathbf{w}_{\text {sat }} / \\
\mathbf{g ~ g}^{-1}\end{array}$ & $\begin{array}{l}\boldsymbol{\theta}_{\mathrm{cc}} / \\
\mathrm{m}^{3} \mathrm{~m}^{-3}\end{array}$ & $\begin{array}{l}\theta_{\mathrm{pm}} / \\
\mathbf{m}^{3} \mathbf{m}^{-3}\end{array}$ & $\begin{array}{l}\rho_{\mathrm{ap}} / \\
\mathrm{g} \mathrm{cm}^{-3}\end{array}$ & $\begin{array}{l}\text { EG } \\
\text { (\%) }\end{array}$ & $\begin{array}{l}\text { CCE } \\
\text { (\%) }\end{array}$ & $\begin{array}{l}\text { Yeso } \\
\text { (\%) }\end{array}$ & $\begin{array}{l}\mathrm{pCO}_{2 \mathrm{p}} \\
/ \mathrm{atm}\end{array}$ & $\begin{array}{l}\mathrm{pCO}_{2 \mathrm{~s}} \\
/ \text { atm }\end{array}$ \\
\hline 1 & 0 & 10 & & 88 & 92 & 1,2 & 0 & 40 & & & $T$ \\
\hline 2 & 10 & 30 & 479 & 94 & 7 & 1, & 0,0 & 0 & 01 & 1 & $10^{-1,97}$ \\
\hline 3 & 30 & 65 & 0,522 & 0,404 & 0,229 & 1,33 & 0,0 & 44 & 0,1 & $10^{-2,43}$ & $10^{-1,47}$ \\
\hline
\end{tabular}

*№: número de capa de suelo; Lím. Sup.: límite superior de la capa; Lím. Inf.: Límite inferior de la capa; $w_{\text {sat }}$ : humedad gravimétrica a saturación; $\theta_{\mathrm{cc}}$ : humedad volumétrica a capacidad de campo; $\theta_{\mathrm{pm}}$ : humedad volumétrica en el punto de marchitez permanente; $\rho_{a p}$ : densidad aparente; EG: elementos gruesos $\left(\varnothing>2 \mathrm{~mm}\right.$ ); CCE: carbonato cálcico equivalente; $\mathrm{pCO}_{2 p}$ : presión parcial de $\mathrm{CO}_{2}$ en equilibrio con la pasta saturada del suelo; $\mathrm{pCO}_{2 s}$ : presión parcial de $\mathrm{CO}_{2}$ en equilibrio con la solución del suelo.

Tabla 7. Calidad promedio del agua del río Segura en el Azud de Alfeitamí*

\begin{tabular}{|c|c|c|c|c|c|c|c|c|c|}
\hline $\begin{array}{l}{\left[\mathrm{Na}^{+}\right] /} \\
\mathrm{meq} \mathrm{L}^{-1}\end{array}$ & $\begin{array}{l}{\left[\mathrm{K}^{+}\right] /} \\
\text {meq L }^{-1}\end{array}$ & $\begin{array}{l}{\left[\mathrm{Ca}^{2+}\right] /} \\
{\text { meq } L^{-1}}\end{array}$ & 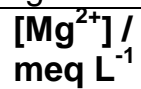 & $\begin{array}{l}{\left[\mathrm{Cl}^{-}\right] /} \\
\text {meq L }^{-1}\end{array}$ & 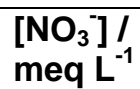 & $\begin{array}{l}{\left[\mathrm{SO}_{4}{ }^{2-}{ }^{-} /\right.} \\
\text {meq L L }^{-1}\end{array}$ & $\begin{array}{l}\text { Alc. / } \\
\text { meq L }^{-1}\end{array}$ & $\mathrm{pH}$ & $\begin{array}{l}\mathrm{CE}_{25}{ }^{\prime} \\
\mathrm{dS}^{-1}\end{array}$ \\
\hline 12,9 & 1,4 & 10,4 & 9,5 & 11,2 & 2,5 & 15,7 & 5,4 & 7,64 & 2,81 \\
\hline
\end{tabular}

Alc.: alcalinidad; $\mathrm{CE}_{25}$ : conductividad eléctrica a $25^{\circ} \mathrm{C}$

\section{Resultados y Discusión}

De acuerdo con las simulaciones llevadas a cabo, en el escenario 1 de implementación del goteo en la jurisdicción de riego JPA Azud de Alfeitamí sin disminución de la dotación de riego, aumentaría la cantidad de agua de riego efectivamente infiltrada en los suelos en dicha jurisdicción, y en consecuencia, la evapotranspiración real y el drenaje (Fig. 4). El incremento de evapotranspiración real se puede cifrar en un $8 \%$ para la sustitución completa del riego por inundación por el localizado manteniendo la dotación. Este aumento de evapotranspiración real significaría una mayor eficiencia de uso del agua por el cultivo y un menor estrés hídrico. El aumento del drenaje, por su parte, se puede cifrar en un $76 \%$ en caso de sustitución total del riego a manta por goteo manteniendo la dotación, y llevaría a una reducción del $10 \%$ en la salinidad de los suelos. Esta menor salinidad daría lugar a un menor estrés salino. Tanto el menor estrés hídrico como el menor estrés salino redundarían en una mayor productividad agrícola siempre que se mantenga la dotación de riego. Como consecuencia del mejor lavado de los suelos la salinidad de los retornos de riego también aumentaría, aunque de manera ligera (Fig. 4). 


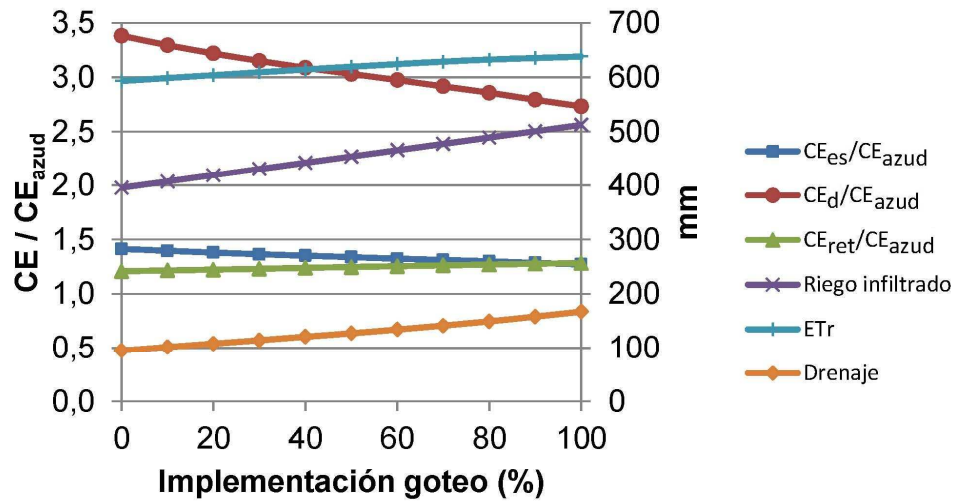

Figura 4. Escenario 1 de implementación del riego por goteo y mantenimiento de la dotación de riego y su efecto en varios parámetros de salinidad e hidráulicos en el JPA Azud de Alfeitamí. Parámetros representados: $\mathrm{CE}_{\mathrm{es}} / \mathrm{CE}_{\mathrm{azud}}$ : salinidad relativa de los suelos respecto del agua del río en el azud; $C E_{d} / \mathrm{CE}_{\text {azud }}$ : salinidad relativa de las aguas de drenaje respecto del agua del río en el azud; $\mathrm{CE}_{\text {ret }} / \mathrm{CE}_{\mathrm{azud}}$ : salinidad relativa de los retornos de riego respecto del agua del río en el azud; Riego infiltrado: lámina de riego efectivamente infiltrada en los suelos; ETr: evapotranspiración real; y drenaje.

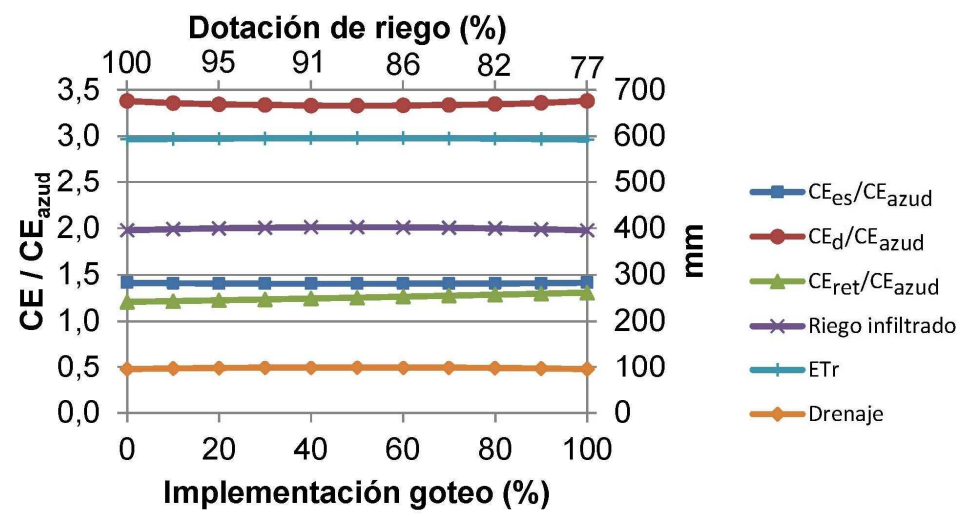

Figura 5. Escenario 2 de implementación del riego por goteo y disminución proporcional de la dotación de riego y su efecto en varios parámetros de salinidad e hidráulicos en la primera jurisdicción (JPA Azud de Alfeitamí). Parámetros representados: ver pie de Fig. 4.

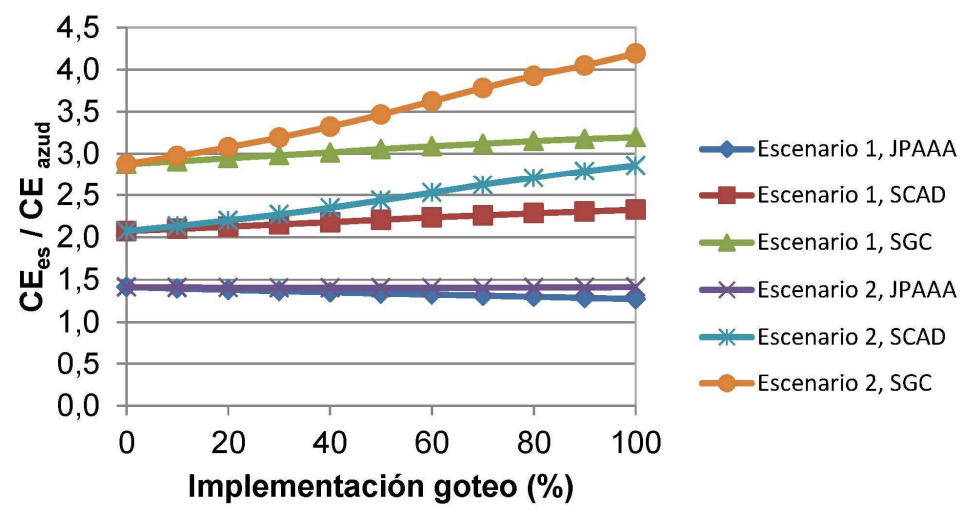

Figura 6. Escenarios 1 y 2 de implementación del riego por goteo en la primera jurisdicción (JPA Azud de Alfeitamí) y cambios resultantes en la salinidad relativa de los suelos de la primera (JPA Azud de Alfeitamí), la segunda (SCA Dolores) y la tercera jurisdicción (SG Carrizales) 
En el escenario 2, más realista, de disminución de la dotación de riego conforme progrese la implementación del riego por goteo, se observa que conforme aumente la eficiencia del sistema de riego, y se reduzca el consumo de agua, la disminución de la dotación anularía todos los beneficios del nuevo sistema. Además, la situación de estrés hídrico y salino no cambiaría respecto de la actual con riego por inundación, y por tanto la salinidad, el riego efectivamente infiltrado, la ETr y los drenajes se mantendrían aproximadamente igual (Fig. 5).

Como consecuencia de la aplicación del escenario 1 en las otras dos jurisdicciones de riego, aumentaría la salinidad de los suelos en la segunda jurisdicción (SCA Dolores), y aún más en la tercera jurisdicción (SG Carrizales). Este efecto ocurriría por el aumento de la salinidad de los retornos de riego de la primera y segunda jurisdicción. Este aumento sería consecuencia del lavado más efectivo de sales en la primera jurisdicción (JPA Azud de Alfeitamí). Esta mayor eficiencia en el lavado se produciría como consecuencia del aumento de la cantidad de agua de riego infiltrada en los suelos en la primera jurisdicción.

Sin embargo, si observamos el escenario 2, más realista que el anterior, con una disminución de la dotación de riego, la implementación del goteo en la primera jurisdicción, no haría variar la salinidad de sus suelos tal y como se ha indicado anteriormente. Por el contrario en las jurisdicciones siguientes la salinidad del suelo aumentaría de forma más acusada que en el escenario 1 de mantenimiento de la dotación de riego. Este efecto resulta de la mayor salinidad de los retornos de riego que van a la segunda y a la tercera jurisdicción.

En el escenario 3 se implementaría el riego por goteo tanto en la primera como en la segunda jurisdicción y se mantendría la dotación de riego en el azud. En este caso la situación descrita para la primera jurisdicción no cambia, y para la segunda jurisdicción se tendría un cierto paralelismo con la primera debido a que en esta segunda aumentaría también la cantidad de agua de riego infiltrada al cambiar el riego a goteo (Fig. 6). Este aumento del agua infiltrada conllevaría un aumento de la evapotranspiración real y el drenaje. El estrés hídrico se reduciría, pero sobre la salinidad de los suelos apenas habría variaciones debido a que hay dos efectos contrapuestos (Fig. 7). Estos son, por un lado el mayor riego infiltrado favorecería el lavado de las sales, pero por otro lado, el agua de riego sería más salina debido al mayor lavado de los suelos de la primera jurisdicción.

En el escenario 4 se implementaría el riego por goteo tanto en la primera como en la segunda jurisdicción disminuyendo proporcionalmente la dotación de riego en el azud. Como consecuencia los parámetros hidrológicos no cambiarían ni en la primera ni en la segunda jurisdicción. Tampoco la salinidad de los suelos en la primera jurisdicción aumentaría (Fig. 5), pero sí lo haría en la segunda de manera más acusada que en el anterior escenario (Fig. 8). Se trata de una consecuencia del aumento de la salinidad de los retornos de riego de la primera jurisdicción (Fig. 5).

En el escenario 5 se implementaría el riego por goteo en todas las jurisdicciones sin cambio de la dotación de riego en el azud. En las jurisdicciones JPA Azud de Alfeitamí y SCA Dolores se tendrían las mismas situaciones descritas en los escenarios 1 y 3 . En la jurisdicción SG Carrizales se tendría un mayor aumento de la salinidad de los suelos y los retornos de riego (Fig. 9).

En el escenario 6 se implementaría el riego por goteo en todas las jurisdicciones con disminución proporcional de la dotación de riego en el azud. En las jurisdicciones JPA Azud de Alfeitamí y SCA Dolores se tendrían las mismas situaciones descritas en los escenarios 2 y 4. En la jurisdicción SG Carrizales se tendría un aumento aún mayor que en el escenario 5 de la salinidad tanto de los suelos como de los retornos de riego. Este último hecho podría 
comprometer también el último uso ecológico de dichos retornos de riego en el Parque Natural de las Salinas de Santa Pola (Fig. 10).

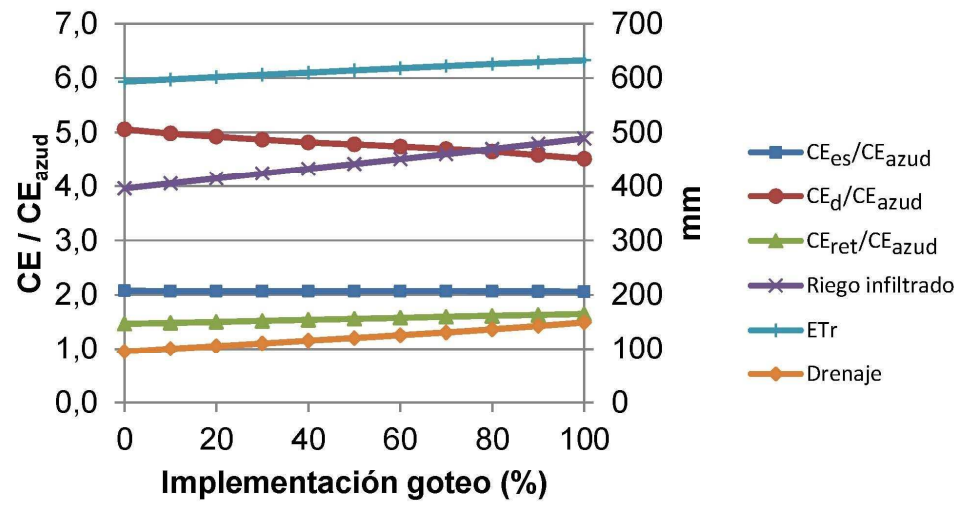

Figura 7. Escenario 3 de implementación del riego por goteo y mantenimiento de la dotación de riego y su efecto en varios parámetros de salinidad e hidráulicos en la segunda jurisdicción (SCA Dolores). Parámetros representados: ver pie de Fig. 4

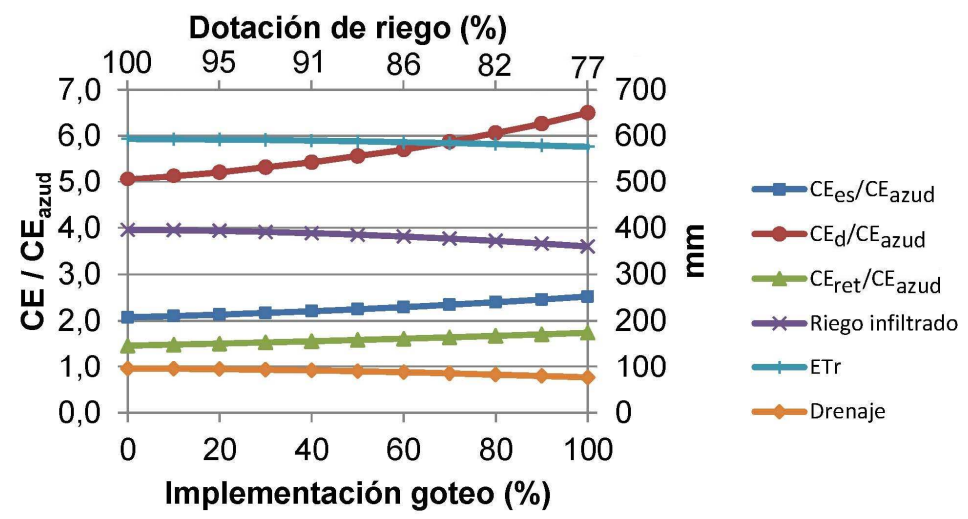

Figura 8. Escenario 4 de implementación del riego por goteo y disminución proporcional de la dotación de riego y su efecto en varios parámetros de salinidad e hidráulicos en la segunda jurisdicción (SCA Dolores). Parámetros representados: Ver pie de Fig. 4

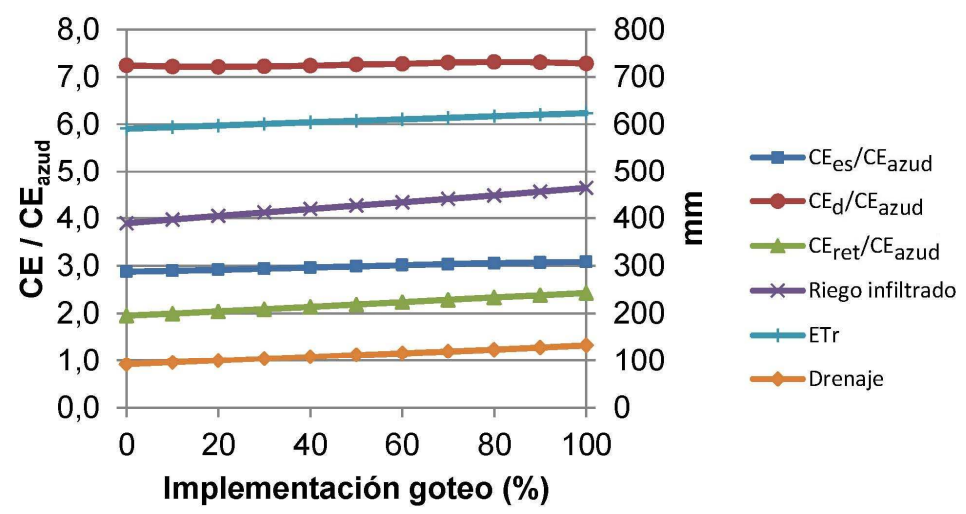

Figura 9. Escenario 5 de implementación del riego por goteo y mantenimiento de la dotación de riego y su efecto en varios parámetros de salinidad e hidráulicos en la tercera jurisdicción (SG Carrizales). Parámetros representados: Ver pie de Fig. 4 


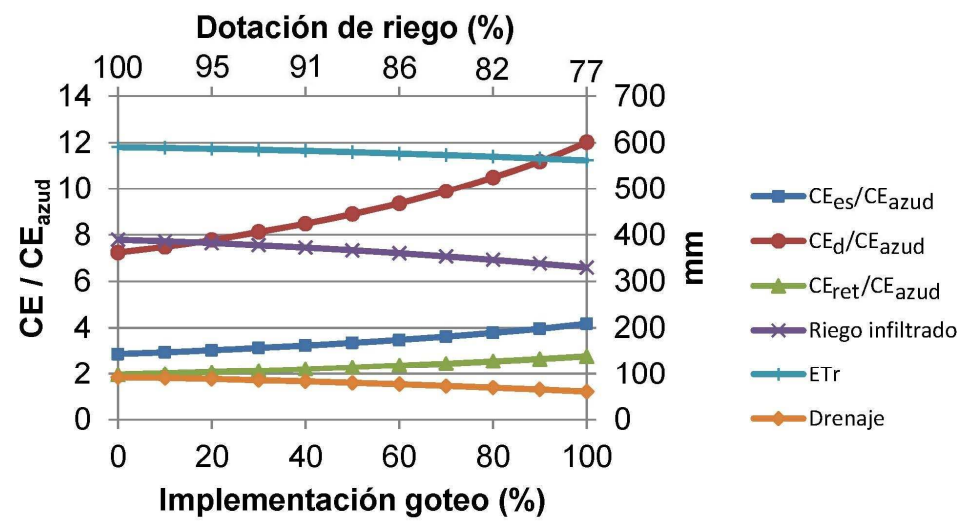

Figura 10. Escenario 6 de implementación del riego por goteo y disminución proporcional de la dotación de riego y su efecto en varios parámetros de salinidad e hidráulicos en la tercera jurisdicción (SG Carrizales). Parámetros representados: Ver pie de Fig. 4

\section{Conclusiones}

El cambio del actual riego por superficie por el riego localizado en la zona tradicional de regadío de la Vega Baja del Segura conllevaría cambios importantes tanto en el funcionamiento hidrológico de toda la zona como en la salinidad de los suelos. Esto se ha puesto de manifiesto mediante la simulación de varios escenarios de modernización del regadío en la parte final de esta zona, la cual está formada por tres jurisdicciones de riego: JPA Azud de Alfeitamí, SCA Dolores y SG Carrizales. Se ha puesto de manifiesto que el cambio del sistema de riego sin alterar la dotación ocasionaría una disminución del estrés hídrico y salino en la primera jurisdicción, apenas cambios en la segunda y un ligero aumento de estos estreses en la tercera. El cambio más profundo ocurriría como consecuencia de la disminución proporcional de la dotación de riego en las tres jurisdicciones. En este caso, en la primera jurisdicción apenas habría cambios, mientras que en la segunda y tercera se presentarían aumentos importantes de la salinidad de los suelos. En consecuencia, se recomienda elaborar un modelo de toda la zona y realizar simulaciones de más escenarios con el objeto de encontrar de qué manera se puede llevar a cabo la modernización del regadío en zona a la vez que se palian al máximo los problemas de salinidad de los suelos y en los retornos de riego.

\section{Bibliografía}

Abadía, R., Ortega, J.F., Ruíz, A. \& García, T. (1999). Análisis de la problemática del regadío tradicional en la Vega Baja del Segura (I): situación actual y consideraciones sobre su modernización. Riegos y Drenajes XXI, 15, 21-31.

Ayers, R.S. \& Westcot, D.W. (1985). Water quality for agriculture. Irrig Drain Paper Paper 29, Rev. 1. Roma, Italia: Food and Agriculture Organization of the United Nations.

Bos, M.G. \& Nugteren, J. (1990). On irrigation efficiencies. Wageningen, Países Bajos: International Institute for Land Reclamation and Improvement (ILRI).

Cavanilles, A.J. (1797). Observaciones sobre la historia natural, geografía, agricultura, población y frutos del Reyno de Valencia. Tomo 2. Madrid, España: Imprenta Real.

CHS. (2015). Plan Hidrológico de la Demarcación del Segura 2015/21. Memoria. Murcia, España: Confederación Hidrográfica del Segura, Ministerio de Agricultura, Alimentación y Medio Ambiente. 
Corwin, D.L., Rhoades, J.D. \& Šimunek, J. (2007). Leaching requirement for soil salinity control: Steady-state versus transient models. Agricultural Water Management, 90, 165-180.

Gea, M. (1997). La formación y expansión decisiva de la Huerta de Murcia-Orihuela. Un enfoque desde la perspectiva de la Orihuela Musulmana (siglos VIII-XIII). Alquibla, 3, 155217.

GVA. (2016). Agricultura destinará 10 millones de euros a la modernización de regadíos en

$$
\text { Vega Baja, }
$$

URL:

http://www.habitatge.gva.es/inicio/area de prensa/not detalle area prensa?id=676222,

Acceso: 5/4/2017.

Melgarejo, J., Trapote, A. \& Roca, J.F. (2013). La infraestructura hidráulica y la gestión del agua en los regadíos tradicionales en la Vega Baja del Segura (Alicante). Revista Pilquén, $13,1-20$.

Rhoades, J.D. (1974). Drainage for salinity control. In: Drainage for Agriculture (ed. J. van Schilfgaarde), pp. 433-461. Soil Science Society of America, Madison (WI, USA).

Rhoades, J.D., Kandiah, A. \& Mashali, A.M. (1992). The use of saline waters for crop production. Roma, Italia: Food and Agriculture Organization of the United Nations (FAO).

Rocamora, C., Abadía, R., Melián, A., Puerto, H. \& Hernández-García, F. (2016). Alternativas de modernización demandadas por los regantes de los regadíos tradicionales de la Vega Baja del Segura. Libro de Actas del XXXIV Congreso Nacional de Riegos (ed. L. Pérez-Urrestarazu). AERYD, Sevilla, España.

Tanji, K.K. \& Kielen, N.C. (2002). Agricultural drainage water management i arid and semiarid areas. Roma, Italia: Food and Agriculture Organization of the United Nations (FAO).

USSL Staff. (1954). Diagnosis and Improvement of Saline and Alkali Soils. Washington D.C: USDA, Agricultural Handbook No. 60.

Visconti, F. \& de Paz, J.M. (2016). DSS-SALTIRSOIL: un sistema de ayuda a la decisión en internet para la obtención de recomendaciones de gestión del riego y el cultivo en tierras por salinización amenazadas. En: Libro de Actas del XXXIV Congreso Nacional de Riegos (ed. L. Pérez-Urrestarazu), pp. 172-181. AERYD, Sevilla, España. 